\title{
Bio-inspired solutions for technical problems: biomimetic cable entries and shock-absorbing pallets
}

\author{
T. Masselter ${ }^{1}$, M. Milwich ${ }^{2}$, H. Monnerat ${ }^{3}$, U. Scharf ${ }^{4}$, \\ M. Hartel ${ }^{4} \&$ T. Speck ${ }^{1}$ \\ ${ }^{I}$ Plant Biomechanics Group Freiburg, Botanic Garden, \\ University of Freiburg, Faculty of Biology, Freiburg, Germany, and \\ Competence Networks Biomimetics and BIOKON e.V. \\ ${ }^{2}$ Institute for Textile and Process Technology in Denkendorf, \\ Denkendorf, Germany \\ ${ }^{3}$ Institute for Technology-Orientientated Design Innovation, \\ Hochschule für Gestaltung, Offenbach am Main, Offenbach/M, Germany \\ ${ }^{4}$ Rittal GmbH \& Co. KG, Herborn, Germany
}

\begin{abstract}
The variability in diameter of organisms or organs (e.g. the ambulacral feet of sea urchins) and fold-flap mechanisms of flowers and leaves served as concept generator for the development of two types of biomimetic cable entry systems. The damping properties of plants and the quills of the hedgehog are the biological models that were translated in a bio-inspired, optimised shockabsorbing transportation pallet. The new cable entry systems are easy to open and to close and are impermeable against fluids or dust. The optimised biomimetic transportation pallet has a very good damping behaviour and is well recyclable.
\end{abstract}

Keywords: biomimetics, cable entry systems, transportation pallets.

\section{Introduction}

A frequently arising problem with conventional cable entry systems is that the cable and the connector plug cannot be fed through the entry system at the same time, because the opening to closed ratio is too small. Exchange of cables with 
connector plugs is relatively time- and, therefore, cost-expensive when using conventional cable entry systems. The new developed biomimetic cable entry systems allows to insert cables together with the plugs and then seal off the cable-plug system effectively against dust and water. These biomimetic structures allow a considerably faster exchange of cables with connector plugs attached.

Delicate goods like computer servers or switch cabinets equipped with highend electronic devices are transported worldwide on shock-absorbing pallets. Standard pallet systems have only limited damping properties and are made of a combination of materials like wood, metal and plastics, making them difficult to recycle. A feasibility study was carried out to analyse if nature can offer role models to create a new pallet, which is more environmental friendly, has better damping and shock absorbing properties and should finally be even cheaper than existing systems.

The process of solving a specific technical problem by investigating biological organisms and translating nature's solutions into technical applications is an example for the "top-down-process" in biomimetics as defined by the Plant Biomechanics Group Freiburg [1], fig. 1 (see also Speck T. \& Speck O. this volume).

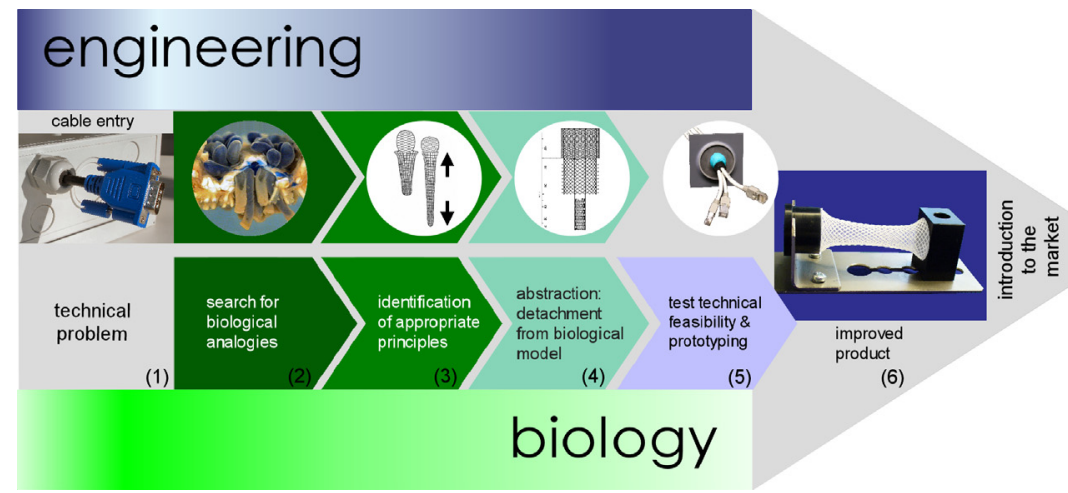

Figure 1: Top-down-process for the development of biomimetic cable-entrysystems.

The starting point for a bionic development in the top-down-process (exemplified here for biomimetic cable entry systems) is the work of the engineer and a technical problem (1). In this case, the low opening-to-closure ratio of most conventional cable entries. Existing technical solutions mainly use panel mounted bases with screw connections and split or shift frames. Conventional cable entry systems providing good impermeability against fluid or dust do not properly address the problem of fast cable exchange. We looked in biology for examples that could be used for solving the technical problem. Different organisms (2), amongst others earthworms and sea urchins were studied and principles were identified that enable the animals to achieve high variability in diameter and form of their body or in different organs (3). Ambulacral feet of sea urchins proved to be very promising concept generators. 
The next step is the abstraction. This means separating the solutions found for the problem from their natural examples (4). This step is decisive for the success of the project. The principles selected for the technical implementation, i.e. the principle of the extensibility of the feet of the sea urchins were translated to technical materials and structures. Following a successful transfer of knowledge from the biologists to the engineers, in a next step, the possibilities for a technical solution have to be considered. While first lab demonstrators were mainly developed in the facilities of the Plant Biomechanics Group Freiburg (5), further potential technical implementations were developed together with the company Rittal and the Institute for Textile and Process Technology in Denkendorf, resulting in optimised demonstrators on pilot plant scale and prototypes (6).

\section{Cable entry systems}

\subsection{Biological templates}

The feet of sea urchins served as concept generators for the first type of cable entry systems, fig. 2A. The tube feet of sea urchins consist of an ambulacral bulb (ampulla) and stem, fig. 2B. Walking takes place by controlled deformation of the feet [2]. Deformation is achieved by contraction of the ambulacral bulb (1), which causes an increase of the volume of liquid in the connected stem (2) of the tube foot. Contractibility and extensibility of the ambulacral bulb and stem is determined by the angle between the collagenous fibres and the longitudinal axis of the foot [3], fig. 2B.

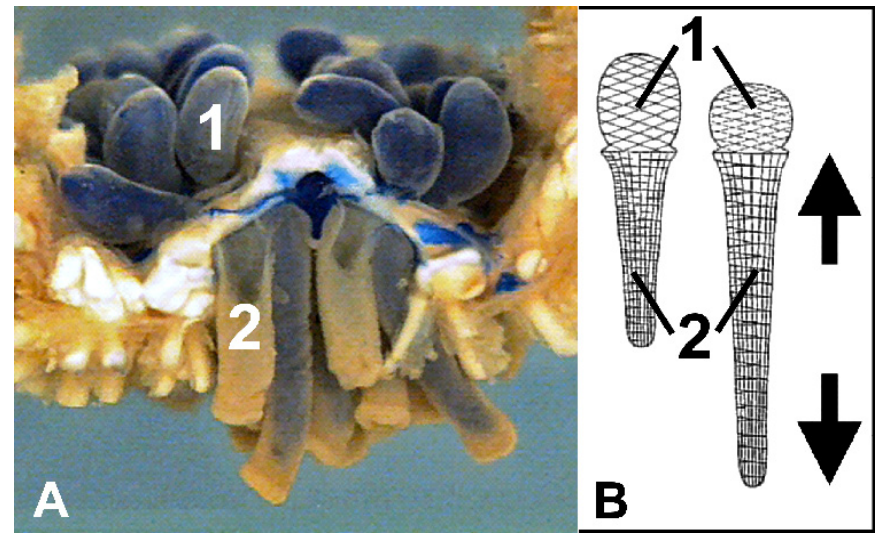

Figure 2: (A) Cross-section through the ambulacral system of a sea urchin showing the ambulacral feet consisting of ampullae (1) and stems (2); from http://biodidiac.bio.uottawa.ca, modified. (B) Schematic drawing of an ambulacral foot showing differing winding angles of the crossed collagenous fibres in the ampulla (1) and the stem (2); from [3], modified. 
A second type of biomimetic cable entries has two biological concept generators: the trapping leaves of the Venus flytrap (Dionaea muscipula) and flowers of the night-blooming cactus Hylocereus undatus, which serves as example of the specialized folding structures in flowers that plants have evolved, fig. 3. While these structures are relatively slow in folding or unfolding, the trapping leaves of the Venus flytrap close very quickly and snap shut. The leaves of the Venus flytrap show a bistable configuration, having the steady states in closed and opened position [4]. A certain amount of force is needed to induce an artificial snapping transition mechanically. In biological reality the fast closure is triggered by insects touching sensor hairs at the inner side of the trapping leaves.
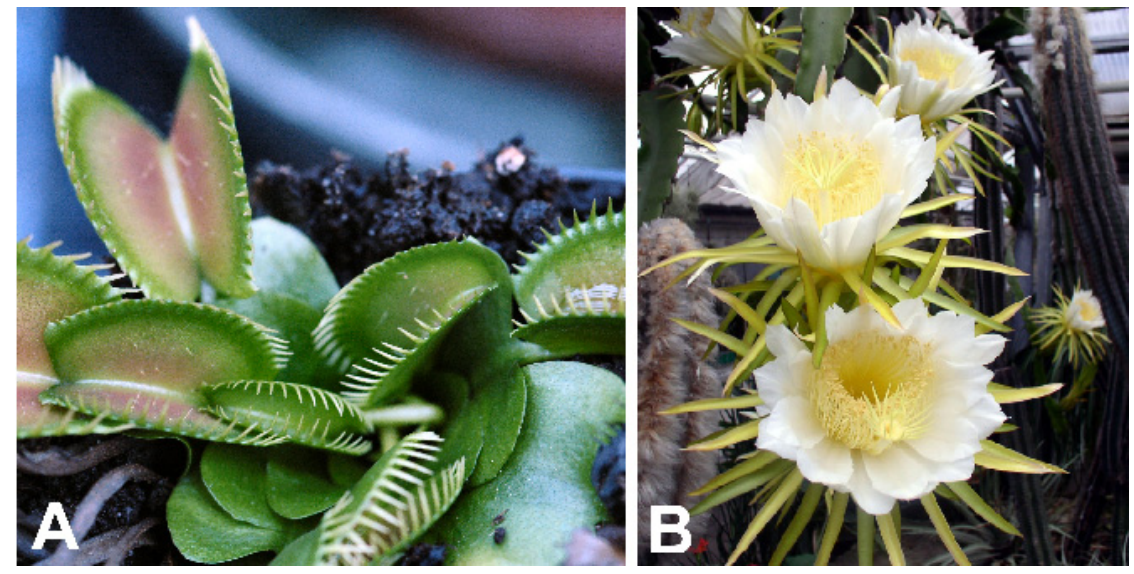

Figure 3: (A) Trapping leaves of the Venus flytrap (Dionaea muscipula). (B) Flowers of the night-blooming cactus Hylocereus undatus; from www.linz.at.

\subsection{Technical translation}

Structure and function of the tube feet of sea urchins were translated into a first type of biomimetic cable entry systems, fig. 4A. With the aid of special braided tubes, a high opening to closed ratio and a high stress relief can be achieved, fig. 4B. These braided tubes consist of helically arranged fibres, and hold similar mechanical properties for extension and contraction than the tube feet of sea urchins. Coating of the braided structures results in a good sealing against dust and fluids.

The closing principles of flowers and trapping leaves were translated into a second type of innovative cable entry systems with a bistable clap-and-fold mechanism, fig. 5. The biomimetic structures are built of a system of interlocking flaps being analogous to the petals and sepals found in flowers. Bistability of the flap-system was inspired by the trapping leaves of the Venus flytrap. The technical structures are easy and fast to open and to close, yet are impermeable to dust and splash-water due to the overlapping structure of the flaps, fig. 5. They also effect a certain stress relief of the cable due to the 

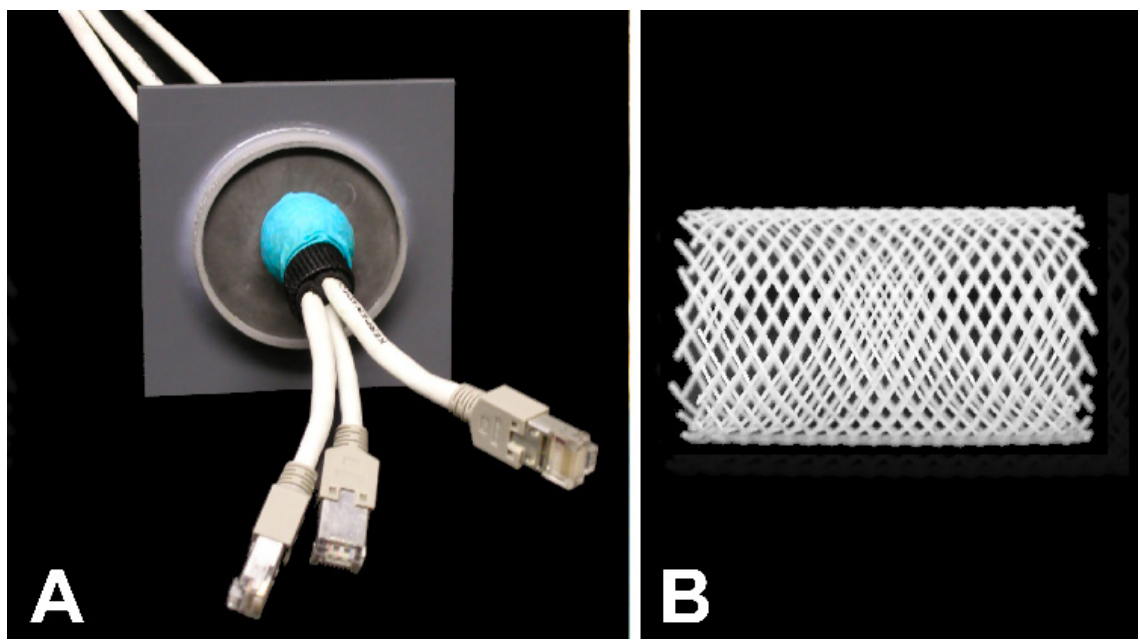

Figure 4: (A) Cable entry system using a coated braided tube (blue). One end of the tube is constantly held open by a panel while the other end is free and can be strained and fixed around the cables. (B) Braided tube made of Nylon-fibres.

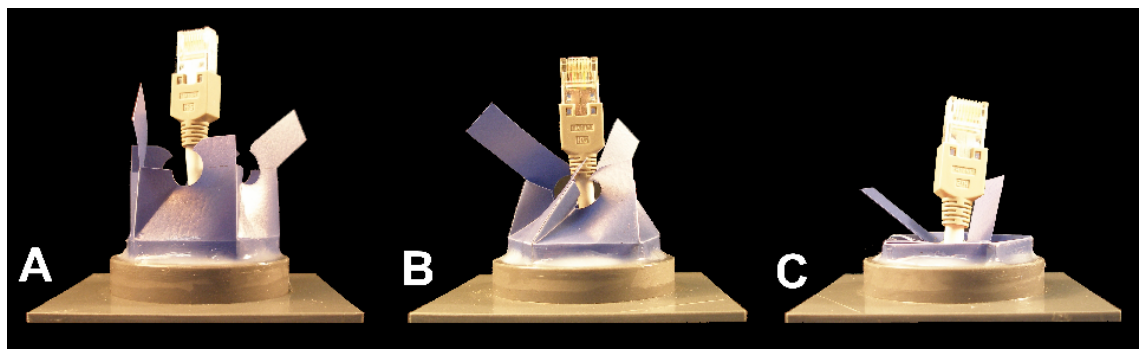

Figure 5: Cable entry system using bistable, interlocking fold-flap structures in opened (A), half-opened (B) and closed (C) state.

bistability of the flaps, since some force is needed to open and close the structures.

\section{Transportation pallets}

\subsection{Biological templates}

In our analysis, we identified several potential biological role models, which could be transferred into a new transportation pallet, figs. 6-8. The biomimetically optimised transportation pallet incorporates damping structures inspired by quills as found in porcupines or hedgehogs (Erinaceus europaeus), fig. 6A. These quills possess radial and longitudinal stiffeners and have good mechanical properties in bending and compression [5-7]. The main function of 

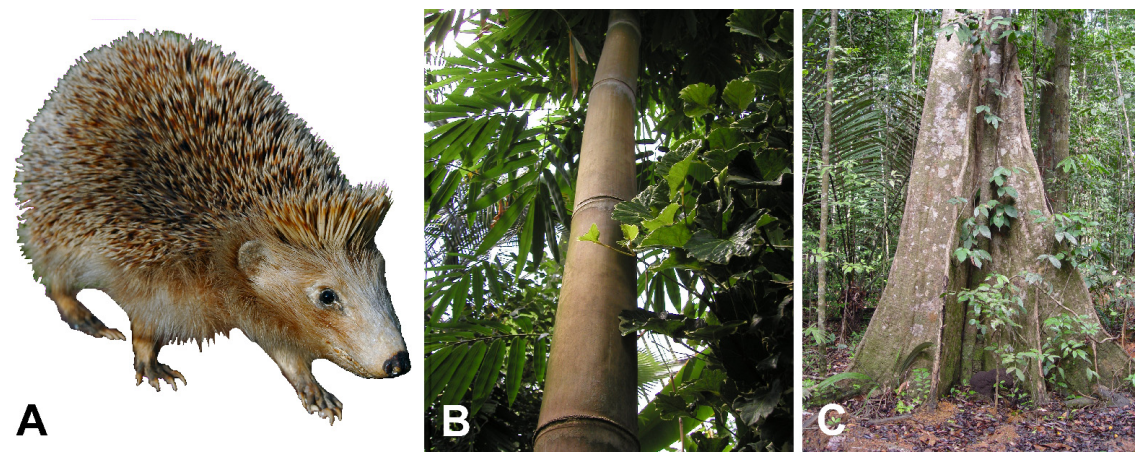

Figure 6: Concept generators for the development of a biomimetically optimised shock-absorbing transportation pallet. (A) West European Hedgehog (Erinaceus europaeus). (B) Stem of the Giant Bamboo (Dendrocalamus giganteus), (C) Buttress (root) of a tropical tree in French Guyana.

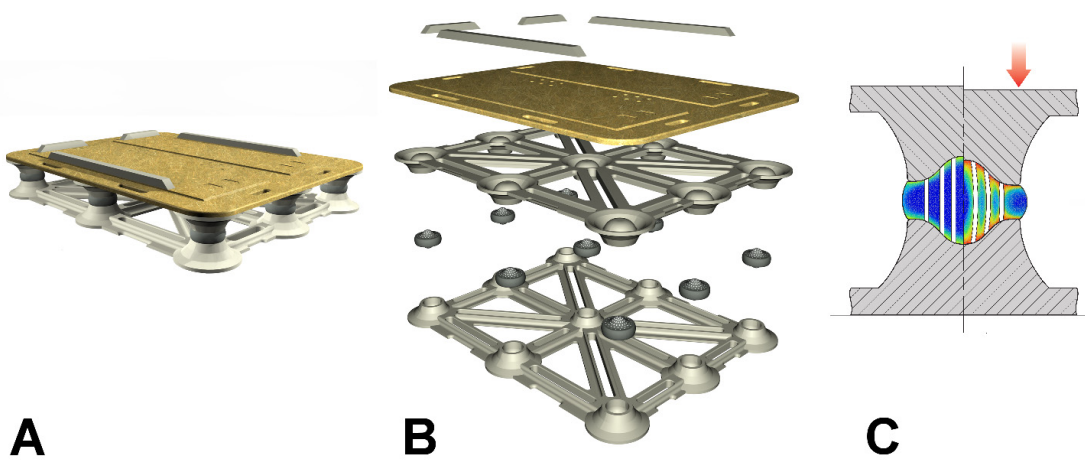

Figure 7: $\quad$ Bio-inspired, optimised shock-absorbing transportation pallet. (A) Complete view of a model of the transportation pallet. (B) Exploded view showing the various elements of the biomimetic pallet. (C) Schematic view of a foot with damping element.

the quills is to protect the animal against predators. In addition, the quills show a significant elasticity and damping when subjected to compressive or bending forces. Climbing porcupines can endure falls from trees without injuries thanks to damping the fall through the elastic bending of the quills.

Other structures with excellent damping behaviour are the stems of the Giant Reed Arundo donax [8] and the culms of bamboo, fig. 6B. The inner structure of the axes of these plants contributes considerably to the overall damping of dynamic loads. The lignified fibre bundles in the stem walls of bamboo and of the Giant Reed show a gradual transition in stiffness to the surrounding cellular matrix of the parenchymatous tissue [9-10]. When subjected to bending 


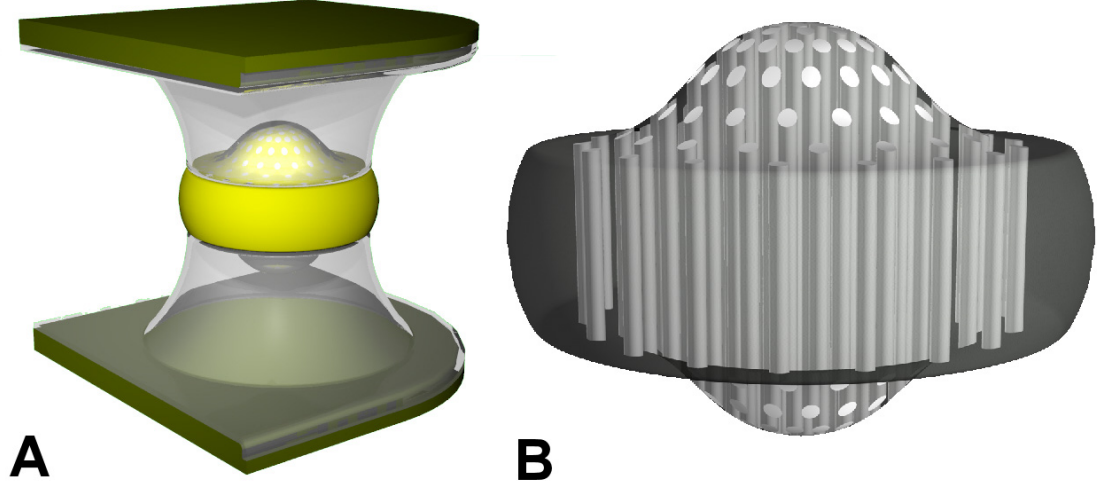

Figure 8: (A) Foot of the transportation pallet with damping element between the two holders. (B) Damping element consisting of rods (light grey) and silicon matrix (dark grey).

vibrations the stems damp a considerable amount of the mechanical energy in each oscillation cycle through shear processes between fibre bundles and cellular matrix [8].

With the help of the special form of the root spur (buttress), trees bridge and diffuse the sharp-cornered notches which they form with the rooted ground surface [11], fig. 6C. On the windward side, thanks to this shape, the stress state along the surface of a tree stem remains (nearly) uniform also in the transition zone between the upright stem and the horizontally arranged roots.

\subsection{Technical translation}

Based on these biological templates, a biomimetic shock-absorbing pallet has been developed. The top and base plates of this transportation pallet consist of fibrous compound materials based on natural fibres (e.g. hemp, flax, linen) and a biomatrix system (e.g. starch, lignin, polylactide acid), thus making both plates fully recyclable, fig. 7 .

The form of the feet, representing the main damping elements of the pallet, has been developed to follow so-called 'baud-curves', which are optimised curvatures avoiding notch-stresses, fig. 7B,C and fig. 8A. These curves are found in many natural structures [12]. The feet consist of two structural components which give them their very good damping behaviour. The 'stiff' damping elements in the feet are rods made of fibrous compound material, fig $7 \mathrm{C}$ and fig. $8 \mathrm{~B}$. They were inspired by the quills of the porcupine, and by the fibre bundles in the stem walls of bamboo, fig. 6B. These rods are embedded in a silicon matrix, representing the 'flexible' damping element of the feet. The silicon matrix is inspired by the parenchymatous matrix embedding the fibre bundles in the bamboo stems. By varying the stiffness of the rod and/or the silicon matrix, different types of feet with different damping behaviour and loadbearing capacities can be produced. The damping feet can be reused many times. 
Considerable amounts of material and energy can thus be saved as well as achieving a reduction of the amount of waste. Therefore, the biomimetic transportation pallet is, in addition to its very good mechanical properties, a considerable contribution to a sustainable development and a reduction of the environmental impact.

\section{Acknowledgements}

The authors would like to thank Antoine Morin from the University of Ottawa and Friedrich Schwarz from The Botanic Garden of Linz for their kind permission to use, respectively, the images of the sea urchin and of the flowers of the night-blooming cactus.

\section{References}

[1] Speck, T., Harder, D. \& Speck, O., Gradient materials and self-repair: learning technology from biology. VDI-Report, B 4284, pp. 1-13, 2007.

[2] Leddy, H.A. \& Johnson, A.S., Walking versus breathing: mechanical differentiation of sea urchin podia corresponds to functional specialization. Biological Bulletin, 198(1), pp. 88-93, 2000.

[3] Wainwright, S.A., Biggs, W.D., Currey, W.D. \& Gosline, J..M., Mechanical Design in Organisms, Princeton University Press: Princeton, 1982.

[4] Forterre, Y., Skotheim, J.M., Dumais, J. \& Mahadevan, L., How the Venus flytrap snaps. Nature, 433, pp. 421-425, 2005.

[5] University of Bath, Department of Mechanical Engineering, www.bath.ac. uk/mech-eng/design-exhibition/pdf/2006DesignExhibitionBooklet.pdf, 08.03.2008.

[6] Dawson, M.A. \& Gibson, L.J., Biomimetics: extending nature's design of thin-wall shells with cellular cores. Design and Nature III, ed. C.A. Brebbia, WIT Press: Southampton, pp. 145-155, 2006.

[7] Karam, G.N. \& Gibson, L.J., Biomimicking of animal quills and plant stems; natural cylindrical shells with foam cores. Material Science and Engineering C2, pp. 113-132, 1994.

[8] Speck, O. \& Spatz, H-C., Damped oscillations of the Giant Reed Arundo donax (Poaceae). American Journal of Botany, 91(6), pp. 789-796, 2004.

[9] Spatz, H.-C., Beismann, H., Brüchert, F., Emanns, A. \& Speck, T., Biomechanics of the giant reed Arundo donax. Philosophical Transactions of the Royal Society London, B 352, pp. 1-10, 1997.

[10] Milwich, M., Speck, T., Speck, O., Stegmaier, T. \& Planck, H., Biomimetics and technical textiles: solving engineering problems with the help of nature's wisdom. American Journal of Botany, 93(10), pp.1295-1305, 2006.

[11] Mattheck, C., Secret Design Rules of Nature. Optimum shapes without computers, Forschungszentrum Karlsruhe GmbH: Karlsruhe, 2007.

[12] Mattheck, C., Engineering components grow like trees. Materialwissenschaften und Werkstofftechnik, 21(4), pp. 143-168, 1990. 